\title{
Charge transport in epitaxial barium titanate films
}

\author{
M. Tyunina ${ }^{1,2,{ }^{*}}$ and M. Savinov ${ }^{2}$ \\ ${ }^{1}$ Microelectronics Research Unit, Faculty of Information Technology and Electrical Engineering, University of Oulu, \\ P. O. Box 4500, FI-90014 Oulu, Finland \\ ${ }^{2}$ Institute of Physics of the Czech Academy of Sciences, Na Slovance 2, 18221 Prague, Czech Republic
}

(Received 28 November 2019; revised manuscript received 28 February 2020; accepted 10 March 2020; published 23 March 2020)

\begin{abstract}
The electrical conductivity of epitaxial $\mathrm{BaTiO}_{3}$ films was studied by small-signal impedance spectroscopy at temperatures of $10-720 \mathrm{~K}$ using $\mathrm{Pt}-\mathrm{BaTiO}_{3}-\mathrm{SrRuO}_{3}$ capacitors. The $\sim 150$-nm-thick $\mathrm{BaTiO}_{3}$ films possessed different lattice strains and degrees of oxygen deficiency. A crossover between the low-temperature hopping of small polarons and the high-temperature semiconductor- to metal-type behavior was demonstrated in all films. It was suggested that the small electron polarons originate from self-trapping at $\mathrm{Ti}$ in the stoichiometric tensilestrained film and from trapping at Ti next to the oxygen vacancy in the oxygen-deficient films. The conductionband transport was ascribed to the thermally activated release of the trapped electrons. It was pointed out that the electronic release can mimic the motion of oxygen vacancies, which are actually immobile.
\end{abstract}

DOI: 10.1103/PhysRevB.101.094106

\section{INTRODUCTION}

Barium titanate $\left(\mathrm{BaTiO}_{3}, \mathrm{BTO}\right)$ is an archetypal representative of the $\mathrm{ABO}_{3}$-perovskite oxide ferroelectrics. These materials possess spontaneous polarization and high dielectric permittivity and exhibit strong electromechanical, electrooptical, electrocaloric, and other effects, which enable numerous electronic applications [1-8]. Depending on application, requirements to the charge transport properties of ferroelectrics vary from insulating (e.g., in capacitors) to conducting (e.g., in thermoelectrics) or both concurrently (e.g., in resistive switching). Because undoped ferroelectrics are wide-band-gap insulators, the electrical conductivity is a function of intentional or unintentional heterovalent cationic doping and/or presence of oxygen vacancies, $V_{O}$, therein. In acceptor-doped ferroelectrics, oxygen vacancies form to compensate for the dopant-induced charge imbalance. Owing to the low formation energy of $\mathrm{V}_{\mathrm{O}}$, oxygen vacancies are also created using high-temperature annealing in a reducing atmosphere (vacuum, hydrogen, nitrogen, etc.). There are limits for maximal thermodynamic equilibrium concentrations of dopants and oxygen vacancies in bulk ferroelectric crystals. For instance, the perovskite phase becomes unstable and a transition to the hexagonal phase occurs when the oxygen deficiency $\delta$ reaches $\sim 0.01$ in $\mathrm{BaTiO}_{3-\delta}$ [9]. With increasing the oxygen deficiency from the stoichiometric composition with $\delta=0$ to that with $\delta \approx 0.01$, the electronic state of perovskite BTO changes from the insulating to an $n$-type conducting state and a metal state [10-12]. Although the influence of oxygen vacancies on the conductivity of BTO is widely acknowledged, the exact mechanism of the charge transport is still under debate. In particular, either electron polarons or conduction-band electrons were and continue to be discussed

*marina.tjunina@oulu.fi as the charge carriers [10-16]. Additionally, oxygen vacancies themselves were suggested to be the mobile charge carriers [17]. The concept of the mobile oxygen vacancies is now widely accepted [18-25].

We note that besides oxygen vacancies, the cationic and/or anionic dopants (e.g., hydrogen), the boundaries between grains and/or with electrodes, and the injected excess carriers can alter the magnitude and character of the conductivity, which makes it difficult to precisely establish the role of oxygen vacancies in the charge transport.

Compared to bulk BTO crystals, different concentrations of oxygen vacancies can be in situ introduced in singlecrystal-type epitaxial films by varying the pressure of ambient oxygen during the films' deposition [24,26-30]. Such films are free from unintentional anionic doping, which may occur during high-temperature treatment in the hydrogen or nitrogen atmosphere. Because the formation energy is the lowest for oxygen vacancies compared to metal vacancies in BTO [31], there is a broad range of oxygen pressures at which oxygen vacancies are formed without changes in the $\mathrm{Ba} / \mathrm{Ti}$ ratio. Additionally, the presence of substrate-imposed biaxial in-plane strain can affect the formation energy for oxygen vacancies in cube-on-cube-type epitaxial films [32-34]. Besides the remarkable possibility to control the concentration of oxygen vacancies in epitaxial films, reducing the film's thickness to $\sim 100 \mathrm{~nm}$ in a vertical electrode-film-electrode capacitor stack enables direct accurate measurements of the conductivity down to $\sim 10^{-14} \mathrm{~S} / \mathrm{cm}$. The impedance of the capacitor can be measured using a small amplitude of the probing ac voltage, which eliminates an injection of the excess carriers. Thus thin epitaxial BTO films enable an excellent opportunity to carefully examine the charge transport and disentangle the electronic, polaronic, or oxygen-vacancy-related processes therein.

Here, we aim to better clarify the role of oxygen vacancies in the charge transport in BTO in general and in epitaxial 
films of BTO in particular. The small-signal impedance spectroscopy was applied to epitaxial 150 -nm-thick perovskite BTO films, sandwiched between the bottom $\mathrm{SrRuO}_{3}$ and top Pt electrodes. The cube-on-cube-type BTO films were grown on $(001) \mathrm{SrTiO}_{3} / \mathrm{SrRuO}_{3}$ by pulsed laser deposition using oxygen pressures of $0.1-20 \mathrm{~Pa}$, which resulted in different in-plane strains and degrees of oxygen deficiency in BTO. The out-of-plane impedance measurements were performed at the frequencies of $1-10^{6} \mathrm{~Hz}$ during thermal cycling between 10 and $720 \mathrm{~K}$. The results were analyzed using an equivalentcircuit modeling and the common conductivity models.

The low-temperature hopping of small polarons and the high-temperature semiconductor- to metal-type conductivity were demonstrated in the films. Trapping of electrons and their thermally activated release were shown to be responsible for the polaronic and the conduction-band transport, correspondingly. The electronic release was argued to imitate the motion of oxygen vacancies, which are immobile de facto.

\section{EXPERIMENT}

Cube-on-cube-type epitaxial BTO films and bottom $\mathrm{SRuO}_{3}$ (SRO) electrode layers were grown on (001) $\mathrm{STiO}_{3}$ substrates by pulsed laser deposition. The oxygen content in BTO was varied by varying the pressure $p_{\mathrm{O} 2}=0.1-20 \mathrm{~Pa}$ of oxygen ambience during the BTO deposition and postdeposition cooling. For electrical characterization, capacitor stacks were formed using the bottom SRO electrode and the circular top Pt contact pads (area $0.2 \mathrm{~mm}^{2}$ ), created by roomtemperature vacuum pulsed laser deposition of $\mathrm{Pt}$ through a shadow mask. The detailed analyses of the films' composition, crystal structure, microstructure, and ferroelectric and optical properties can be found elsewhere [35,36].

Compared to the room-temperature pseudocubic unit cell of bulk BTO [lattice parameter $a_{0}=V_{0}^{1 / 3}=\left(a_{\mathrm{BTO}}^{2} c_{\mathrm{BTO}}\right)^{1 / 3}$ ], the films experience the in-plane biaxial strain $\left(s_{a}=a / a_{0}-1\right)$ and unit-cell expansion $\left(\Delta V / V_{0}=V / V_{0}-1\right)$ (Table I). The expansion agrees with the strain and elastic properties of BTO for the stoichiometric $20-\mathrm{Pa}$ film, but it opposes the estimated elastic shrinkage and indicates the presence of oxygen vacancies in the 0.1-10-Pa films. Because of the combined action of epitaxial strain and oxygen vacancies, the expansion $\Delta V / V_{0}$ is not proportional to the vacancy concentration but characterizes it qualitatively [32-34]. The films in-plane and out-of-plane lattice parameters ( $a$ and $c$ ) and tetragonality $(c / a-1)$ imply that the low-pressure $0.1-5-\mathrm{Pa}$ films adopt

TABLE I. The room-temperature in-plane biaxial strain $s_{a}$, unitcell expansion, tetragonality $(c / a-1)$, and type of the ferroelectric phase, and the paraelectric phase transition temperature $T_{\mathrm{PE}}$ in the films deposited at different oxygen pressures $p_{\mathrm{O} 2}$.

\begin{tabular}{lccccc}
\hline \hline$p_{\mathrm{O} 2}(\mathrm{~Pa})$ & 0.1 & 2 & 5 & 10 & 20 \\
\hline$s_{a}(\%)$ & -0.04 & -0.19 & -1.71 & +0.41 & +0.61 \\
$\Delta V / V_{0}(\%)$ & +3.14 & +2.73 & +1.40 & +1.20 & +1.30 \\
$(c / a-1)(\%)$ & +3.27 & +3.32 & +6.80 & -0.02 & -0.51 \\
FE phase & $c$ & $c$ & $c$ & $a$ & $a$ \\
$T_{\mathrm{PE}}(\mathrm{K})$ & $-{ }^{\mathrm{a}}$ & $\sim 550$ & $\sim 550$ & $\sim 450$ & $\sim 450$ \\
\hline \hline
\end{tabular}

${ }^{\mathrm{a}}$ Large conductivity prevents ferroelectric characterization. a $c$-type ferroelectric phase and the high-pressure 10-20-Pa films adopt an $a$-type ferroelectric phase [37,38]. The phase transition to the paraelectric state occurs at the temperatures $T_{\mathrm{PE}}>400 \mathrm{~K}$.

The impedance of the capacitors was measured by a Novocontrol Alpha-AN high performance frequency analyzer. As a probing small signal, we used the sinusoidal ac voltage with the amplitude of $1-10 \mathrm{mV}$ and the frequency $f=$ $\left(1-10^{6}\right) \mathrm{Hz}$. The voltage was applied and the response was measured along the out-of-plane direction of the capacitors. The impedance was analyzed using an equivalent-circuit model of a leaky parallel-plate capacitor [39-42].

Temperature control was realized using a Janis ST-100 He flow cryostat equipped with a Lake Shore 335 temperature controller. The temperature was swept at a rate of $3-5 \mathrm{~K} / \mathrm{min}$. The measurements were performed during preliminary cooling to a low temperature $T_{\min }=8-100 \mathrm{~K}$, first initial heating to a high temperature $T_{\max }=400-750 \mathrm{~K}$, cooling after the initial heating, and consequent heating and cooling runs. The measurements were repeated after several weeks, during which the capacitors were kept at room temperature.

\section{RESULTS}

The impedance of the ferroelectric thin-film capacitor is a function of frequency [40-42]. Correspondingly, the capacitance $C(f)$, extracted from the measured impedance, exhibits frequency dispersion at all temperatures (Fig. 1). This shape of the $C(f)$ plots is well simulated using the equivalentcircuit model [39-42], which includes the capacitance $C$ and the resistance $R_{F}$ of the film and the resistance $R_{E}$ of the electrodes (inset in Fig. 1 and Figs. S1 and S2 in the Supplemental Material [43]). The film's resistance comprises the dielectric-loss fraction $R_{\varepsilon}$ and Ohmic resistance $R_{\Omega}$. As shown before [40-42], a relatively small resistance of the film leads to an increased $C(f)$ at lower frequencies, while a rather small resistance of the electrodes is responsible for the higher-frequency drop of $C(f)$. With increasing temperature, the dispersion $C(f)$ changes primarily due to a progressive decrease of the film's Ohmic resistance $R_{\Omega} \ll R_{\varepsilon}$.

During the first initial heating of the virgin films, we observe very high peaks in $C(f, T)$ at $T \geqslant 600 \mathrm{~K}$ in the

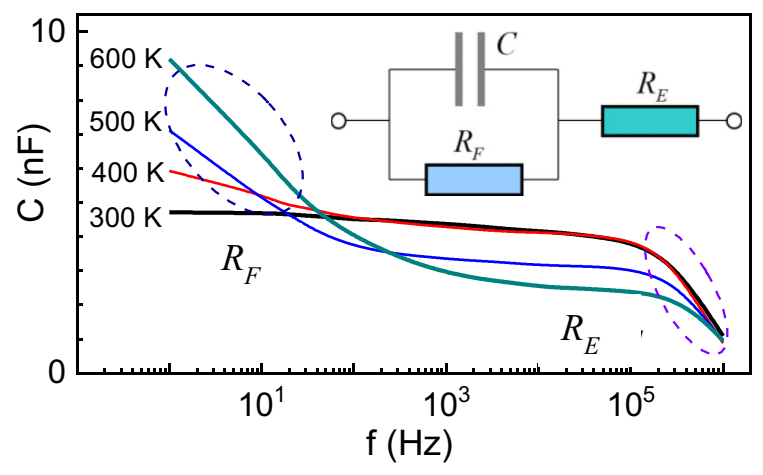

FIG. 1. Frequency dispersion of the measured capacitance at different temperatures for the $20-\mathrm{Pa}$ film. Inset shows equivalent circuit of the ferroelectric thin-film capacitor. Effects of the film resistance $R_{F}$ and electrode resistance $R_{E}$ on the dispersion are marked by dashed circles. 

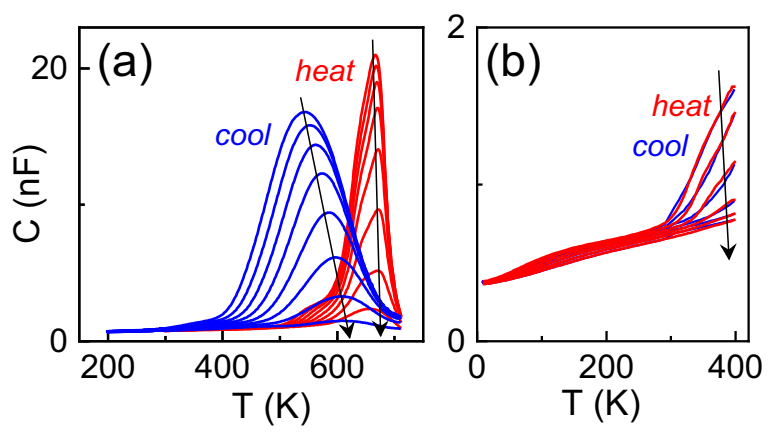

FIG. 2. (a),(b) Capacitance as a function of temperature during initial heating to (a) $710 \mathrm{~K}$ and (b) $400 \mathrm{~K}$ and consequent cooling in the as-deposited 5-Pa BTO film. Arrows show directions of frequency increase from $500 \mathrm{~Hz}$ to $100 \mathrm{kHz}$.

oxygen-deficient 2-10-Pa films (Fig. 2(a) and Supplemental Fig. S3 [43]). During the consequent cooling, broad frequency-dependent spreads over the wide temperature range $T>300-400 \mathrm{~K}$ are found (Fig. 2(a) and Supplemental Fig. S1 [43]). During the further heating and cooling runs, the behavior continues to be like that during the cooling after the initial heating. For convenience, here, we denote the initial and consequent behaviors as "virgin" and "excited." The initial virgin state and behavior are recovered after several weeks of storing the films at room temperature. Importantly, the peaks and thermal hysteresis occur only when the very high temperature $T \geqslant 600-700 \mathrm{~K}$ is reached, while the behavior is hysteresis free during the heating-cooling runs in the range of lower temperatures [Fig. 2(b)]. At the absence of oxygen deficiency, in the 20-Pa films, the peculiar high-temperature peak and thermal hysteresis are absent (Supplemental Fig. S3 [43]). In the 0.1-Pa film, the large conductivity $\sigma$ prevents capacitance measurements. During the first heating of the virgin state, the paraelectric phase transitions are clearly manifested by the capacitance and inverse-capacitance peaks at $\sim 450 \mathrm{~K}$ in the 10- and 20-Pa films (Supplemental Fig. S3 [43]). In the 2- and 5-Pa films, the low-temperature Curie-Weiss behavior evidences the ferroelectric state at $<500 \mathrm{~K}$ with the phase transition temperature of approximately $\geqslant 550 \mathrm{~K}$, whose more precise detection is hindered by large conductivity (Supplemental Fig. S3 [43]).

The character of the dispersion $C(f)$ suggests a hightemperature growth of the BTO conductivity. Indeed, the determined ac conductivity $\sigma$ increases with temperature and exhibits thermal hysteresis (Fig. 3). During the initial virgin heating, a steep increase of the lower-frequency $\sigma$ at the temperatures $T \geqslant 500-600 \mathrm{~K}$ is observed in all films except the $0.1-\mathrm{Pa}$ film. This increase confirms that the high-temperature capacitance peaks are primarily due to the dramatic reduction of the films' resistance. Interestingly, a peculiar drop in the conductivity is seen at approximately $500 \mathrm{~K}$ in the $2-10-\mathrm{Pa}$ films [Figs. 3(b)-3(d)] and is weakly expressed in the 20-Pa film [Fig. 3(e)].

The temperature evolution of the conductivity during the virgin heating is analyzed for the frequency of $1 \mathrm{~Hz}$, at which the ac conductivity, $\sigma_{0}$, is approximately equal to the $\mathrm{dc}$ conductivity (Fig. 4 and Supplemental Fig. S4 [43]). There are three distinct temperature ranges with different types of behavior $\sigma_{0}(T)$ : low temperatures $T<250 \mathrm{~K}$ (LT), medium temperatures $250 \mathrm{~K}<T<450-500 \mathrm{~K}$ (MT), and high temperatures $T>500 \mathrm{~K}$ (HT) [Fig. 4(a)]. The magnitude of $\sigma_{0}$ is significantly smaller than $10^{-8} \mathrm{~S} / \mathrm{cm}$ in the LT and MT ranges for the 2-20-Pa films, which classifies these films as insulators. In the $0.1-\mathrm{Pa}$ film, the magnitude of $\sigma_{0}$ is insulatorlike in the LT range and semiconductorlike at higher temperatures.

For the strongly oxygen-deficient $0.1-\mathrm{Pa}$ film in the LT range [Fig. 4(b)], the small values of $\sigma_{0} \approx 10^{-10}-10^{-8} \mathrm{~S} / \mathrm{cm}$
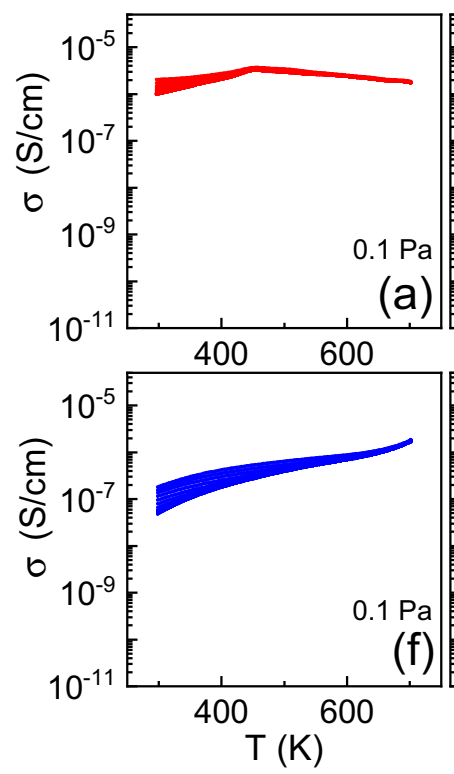
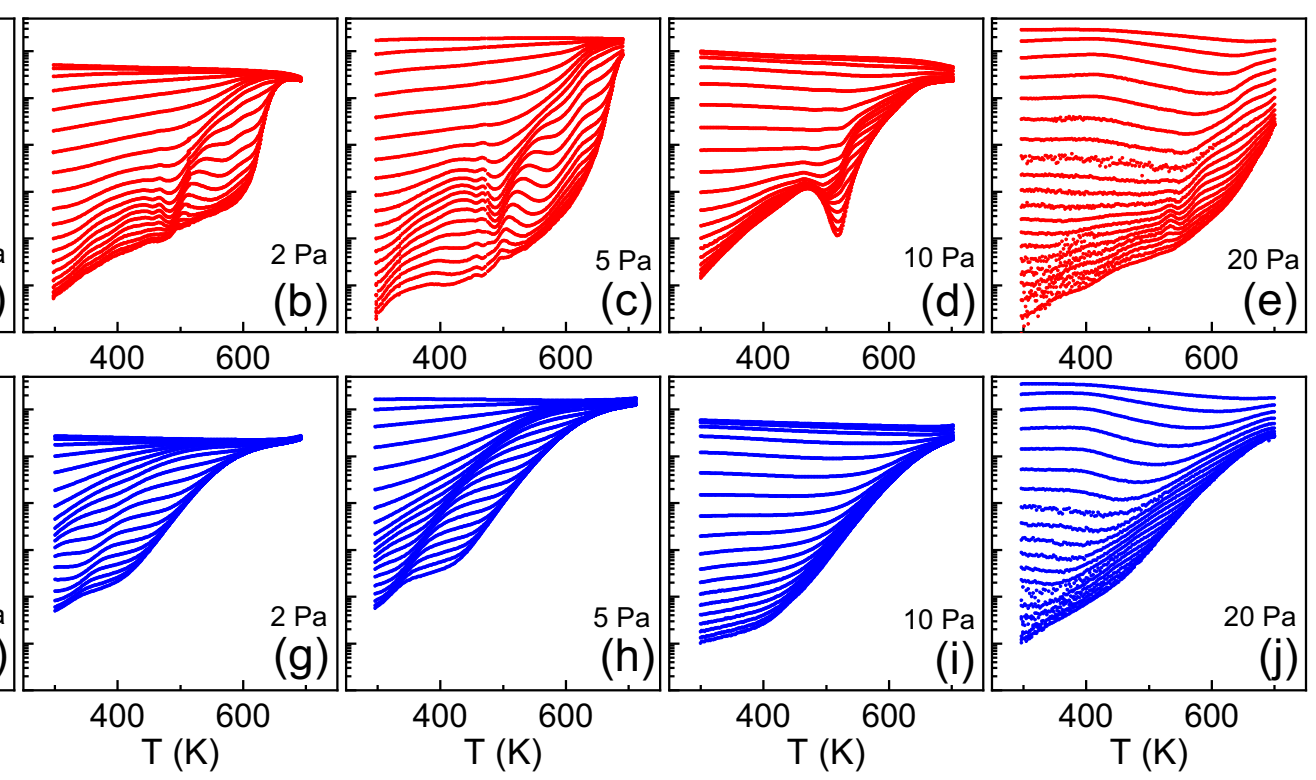

FIG. 3. Conductivity as a function of temperature during (a)-(e) heating and (f)-(j) consequent cooling in the BTO films deposited at different oxygen pressures. Frequencies are from $10 \mathrm{~Hz}$ to $1 \mathrm{MHz}$ (from the lower curves up). The deposition pressures are marked on the plots. 

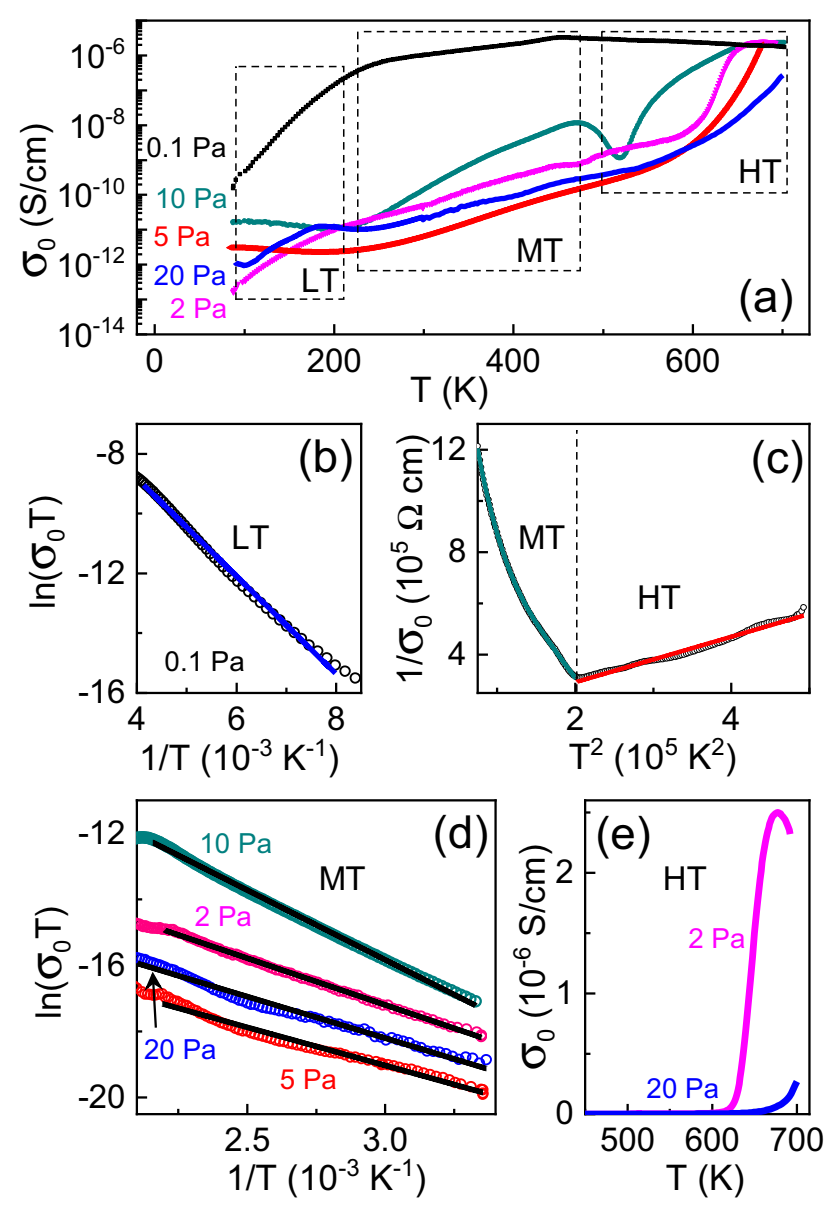

FIG. 4. (a) Conductivity as a function of temperature during the first heating. Frequency is $f=1 \mathrm{~Hz}$. Different specific temperature regions are marked by dashed squares. (b),(c) Conductivity-temperature relationships for (b) polaronic hopping and (c) semiconductor- and metal-type behavior in the 0.1-Pa film. Solid lines show fits. (d),(e) Conductivity-temperature relationships in the 2-20-Pa films: (d) polaronic hopping and (e) tendency to metal-type behavior. Solid lines show fits in (d).

and a good linear fit $\left[\ln \left(\sigma_{0} T\right) \propto 1 / T\right]$ indicate the insulator state with a polaronic-hopping mechanism of the conductivity $\left[\sigma_{0} \propto T^{-1} \exp \left(-E_{h} / k T\right)\right][13,14]$. Here $E_{h}$ is the hopping energy $E_{h} \approx 0.15 \mathrm{eV}$. In the MT range for this film, the larger magnitude of $\sigma_{0} \approx(1-3) \times 10^{-6} \mathrm{~S} / \mathrm{cm}$ and a power- law increase with temperature point to a semiconductorlike conductivity. In the HT range, a decrease of $\sigma_{0}(T)$ is found. A linear relationship $\left(1 / \sigma_{0} \propto T^{2}\right)$ suggests a metal-like conduction therein [Fig. 4(c)]. A semiconductor-metal transition takes place at approximately $450 \mathrm{~K}$.

For the 2-20-Pa films, the small LT conductivity of $\sigma_{0} \leqslant$ $10^{-11} \mathrm{~S} / \mathrm{cm}$ signifies excellent insulating properties, but, concurrently, makes it difficult to establish a mechanism of the charge transport (if any) because of the polarization relaxation contributing to $R_{F}$. In the MT range, fits to hopping of small polarons with $E_{h} \approx 0.2-0.3 \mathrm{eV}$ are valid [Fig. 4(d)]. In the HT range, the conductivity steeply increases up to a metal-like state in the oxygen-deficient 2-10-Pa films, but this state is not achieved in the stoichiometric 20-Pa film [Figs. 4(a) and 4(e)].

The polaronic hopping is verified by investigations of the frequency dispersion of the ac conductivity $\sigma(f)$ (Fig. 5 and Supplemental Fig. S4 [43]). Good fits to the universal relaxation law $\left[\sigma(f) \propto f^{\gamma}\right]$ are obtained in the LT and MT ranges in the 2-20-Pa films and in the LT range for the $0.1-\mathrm{Pa}$ film (Fig. 5). The fitted exponent is $\gamma \approx 0.9-1.0$, which confirms barrier hopping of the small polarons therein $[44,45]$. Interestingly, the behavior of $\sigma(f)$ is peculiar and cannot be fitted by a single relaxation in the vicinity of $\sim 500 \mathrm{~K}$ in the 2-10-Pa films [Figs. 5(b)-5(d)]. This peculiarity is consistent with the relative drop of $\sigma$ at $\sim 500 \mathrm{~K}$ [Figs. 3(b)-3(e)].

The films' conductivity during cooling after the first heating to $\sim 700 \mathrm{~K}$, in the excited state, is shown in (Fig. 6). The $0.1-\mathrm{Pa}$ film is semiconductorlike at high temperatures [Fig. 6(a)]. In the 2-10-Pa films, good fits to $\left[\sigma_{0} T \propto\right.$ $\left.\exp \left(-E_{h} / k T\right)\right]$ with $E_{h} \geqslant 0.8 \mathrm{eV}$ for $T>400 \mathrm{~K}$ [Fig. 6(b)] may be interpreted as hopping of large polarons [18,19,22]. The small polarons are found for $T<400 \mathrm{~K}$ in the 2-20-Pa films and $T<200 \mathrm{~K}$ in the 0.1-Pa film [Fig. 6(c)]. The lowtemperature hopping of the small polarons is confirmed by the behavior $\left[\sigma(f) \propto f^{\gamma}\right]$ with $\gamma \approx 0.9-1.0$ in the $2-20-\mathrm{Pa}$ films [Figs. 6(d) and 6(e)]. A crossover between the low- and high-temperature behavior, which is seen at approximately $\sim 400 \mathrm{~K}$ in $\sigma_{0}(T)$ [Fig. 6(a)], is also manifested by a peculiar two-component frequency dispersion $\sigma(f)$ in the oxygendeficient 2-10-Pa films [Fig. 6(d)].

\section{DISCUSSION}

Thus hopping of the small polarons determines the charge transport in the insulating stoichiometric 20-Pa and

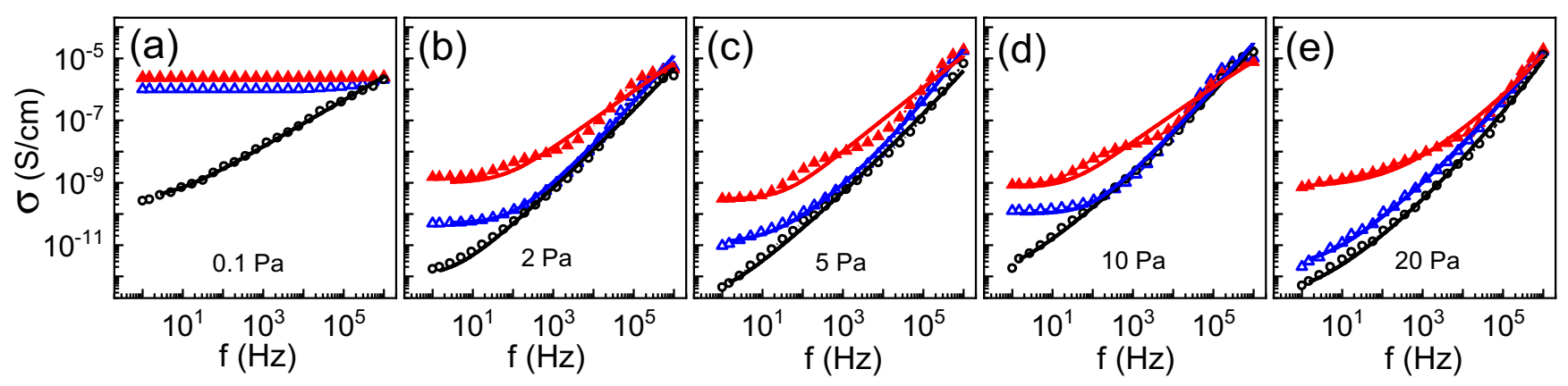

FIG. 5. Ac conductivity as a function of frequency at $T=100,300$, and $500 \mathrm{~K}$ (from lower curves up) determined during the first heating in the films deposited at different pressures. 

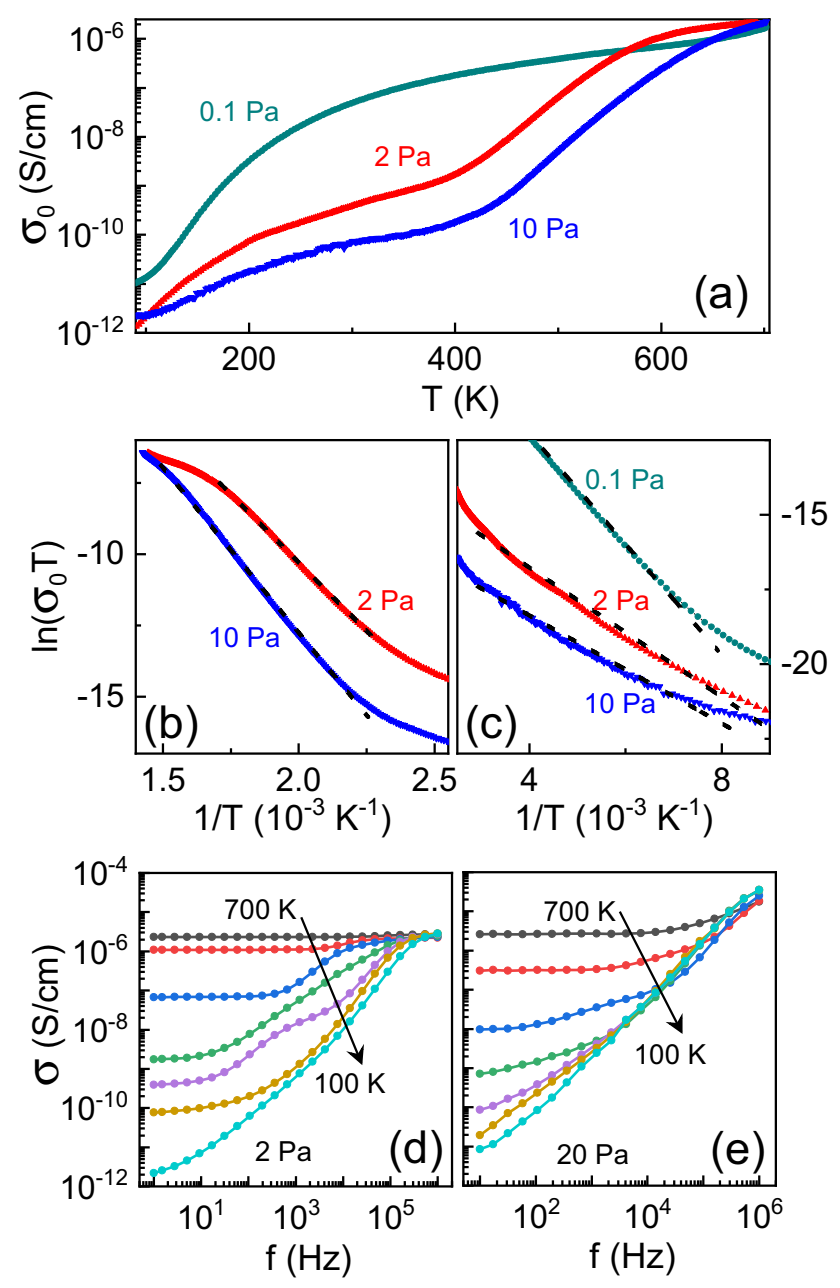

FIG. 6. Cooling after the initial heating. (a) Conductivity $\sigma_{0}$ (at $f=1 \mathrm{~Hz}$ ) as a function of temperature. (b),(c) Conductivitytemperature relationship for polaronic hopping. Dashed lines show fits. (d),(e) Ac conductivity as a function of frequency at $T=700$, $600,500,400,300,200$, and $100 \mathrm{~K}$. Arrows show directions of temperature decrease. Deposition pressures are marked on the plots.

oxygen-deficient $0.1-10-\mathrm{Pa}$ films in the broad range of temperatures below a certain crossover temperature $T_{\sigma}: T<T_{\sigma}$. For the higher temperatures $T>T_{\sigma}$, the thermally activated generation of the free charge carriers results in the massive increase of the conductivity up to the metal-like state during the first heating. During the following consequent thermal cycling, hopping of the small polarons is detected at $T<T_{\sigma}$ and either hopping of the large polarons or semiconductor behavior is observed at $T>T_{\sigma}$. In the vicinity of $T_{\sigma}$, the two conductivity processes coexist, which is manifested by the relative drop of the conductivity $\sigma_{0}(T)$ and the doublecomponent frequency dispersion $\sigma(f)$. The characteristic temperatures $T_{\sigma}$ and activation energies $E_{h}$ depend on the film's thermal history and oxygen deficiency as summarized in Table II. There is no correlation between the crossover temperatures $T_{\sigma}$ and the temperatures $T_{\mathrm{PE}}$ of the ferroelectricto-paraelectric phase transition (Table I).

There have been few theoretical investigations of the formation and structure of oxygen vacancies in epitaxially
TABLE II. The crossover temperatures $T_{\sigma}$ and the activation energies $E_{h}$ in the virgin and excited states in the films deposited at different pressures $p_{\mathrm{O} 2}$.

\begin{tabular}{lccccc}
\hline \hline$p_{\mathrm{O} 2}(\mathrm{~Pa})$ & 0.1 & 2 & 5 & 10 & 20 \\
\hline Virgin & & & & & \\
$T_{\sigma}(\mathrm{K})$ & 250 & 455 & 460 & 475 & 550 \\
$E_{h}(\mathrm{eV})$ & 0.15 & 0.25 & 0.20 & 0.35 & 0.20 \\
Excited & & & & & \\
$T_{\sigma}(\mathrm{K})$ & 230 & 390 & 410 & 420 & 550 \\
$E_{h}(\mathrm{eV})$ & 0.15 & 0.10 & 0.10 & 0.05 & 0.20 \\
$E_{h}(\mathrm{eV})$ & - & 0.80 & 0.85 & 0.95 & - \\
\hline \hline
\end{tabular}

strained BTO films. Here, we qualitatively discuss our observations by extrapolating the knowledge acquired for bulk BTO and $\mathrm{SrTiO}_{3}$ (STO). In BTO and STO, oxygen vacancies $\mathrm{V}_{\mathrm{O}}$ can exist as neutral $\left(\mathrm{V}_{\mathrm{O}}^{0}\right)$, singly positive $\left(\mathrm{V}_{\mathrm{O}}^{+}\right)$, and/or doubly positive $\left(\mathrm{V}_{\mathrm{O}}^{+2}\right)$ ones. The vacancy $\mathrm{V}_{\mathrm{O}}^{+2}$ can trap one to two electrons in the form of small polarons. In such polarons, the electrons are localized on $\mathrm{Ti}$ atoms near the vacancy, which changes $\mathrm{Ti}^{4+}$ to $\mathrm{Ti}^{3+}$. Additionally, an isolated small polaron may occur as an electron localized on a single Ti atom (self-trapped electron polaron). A self-trapped hole polaron localized on an individual $\mathrm{O}$ atom is also possible. According to the recent theoretical analyses [46,47], the lowest formation energy is found for the doubly charged vacancies $\mathrm{V}_{\mathrm{O}}^{+2}$ and the complexes of $\mathrm{V}_{\mathrm{O}}^{+2}$ and one polaron, or $\left(\mathrm{V}_{\mathrm{O}}^{+2}-\mathrm{Ti}^{3+}\right)^{+}$.

It was also shown that the in-plane biaxial strain can affect the formation energy for $V_{O}$ in dependence on the strain sign and the position of $\mathrm{V}_{\mathrm{O}}$ in (001) epitaxial films [32-34]. In particular, the in-plane compressive strain can reduce the formation energy for an out-of-plane vacancy $\mathrm{V}_{O}$ in the Ti-O plane, which is normal to the substrate surface (i.e., in the $\mathrm{Ba}-\mathrm{O}$ plane, which is parallel to the substrate surface). Concurrently, the in-plane compression can raise the formation energy for an in-plane vacancy $\mathrm{V}_{\mathrm{O}}$ (in the Ti-O plane parallel to the substrate surface). Therefore, in the oxygen-deficient in-plane compressed BTO films (see Table I), the out-of-plane oxygen vacancies are preferred and the dipolar complexes $\left(\mathrm{V}_{\mathrm{O}}^{+2}-\mathrm{Ti}^{3+}\right)^{+}$can be out-of-plane oriented. Recently, the presence of such complexes was experimentally detected [36]. Another effect of epitaxial strain was suggested to be in stabilization of the self-trapped electron polarons in BTO [47]. Interestingly, the self-trapping was found to be more efficient for the in-plane tensile strain.

It is likely that the self-trapped electron polarons are primarily responsible for the charge transport at $T<T_{\sigma}$ in the stoichiometric 20-Pa film. This $a$-type film experiences the in-plane biaxial tension, which arises from the relaxation of the BTO-STO misfit strain during the high-temperature film growth and the consequent buildup of the thermal mismatch strain during the postdeposition cooling [35]. The tension may promote the self-trapping [48]. The self-trapped polarons are also possible in the $a$-type virgin 10-Pa film. With increasing oxygen deficiency (decreasing deposition pressure $p_{\mathrm{O} 2}$ ), the $c$ type tetragonal films and the $\left(\mathrm{V}_{\mathrm{O}}^{+2}-\mathrm{Ti}^{3+}\right)^{+}$complexes form (Table I). Hence, the electron polarons are mainly those next to the oxygen vacancy therein. These polarons determine the 
low-temperature conductivity in the $0.1-5-\mathrm{Pa}$ films and may contribute to that in the $10-\mathrm{Pa}$ film.

We note that the trapping and binding energy of polarons depend on strain and polarization [47]. Here, the lattice strain and, hence, polarization are not monotonic functions of oxygen pressure (Table I). Therefore, the concentration and activation energy of polarons and the polaronic conductivity are not monotonic functions of oxygen pressure either (Fig. 4 and Table II).

Considering the out-of-plane oriented $\left(\mathrm{V}_{\mathrm{O}}^{+2}-\mathrm{Ti}^{3+}\right)^{+} \mathrm{de}-$ fects as dominating, the profound high-temperature increase of the conductivity in the virgin films can be ascribed to a thermally activated release of the electrons from $\left(\mathrm{V}_{\mathrm{O}}^{+2}-\mathrm{Ti}^{3+}\right)^{+}$ to the conduction band. During the initial heating, first, the concentration of polarons diminishes as the electrons start escaping from the $\left(\mathrm{V}_{\mathrm{O}}^{+2}-\mathrm{Ti}^{3+}\right)^{+}$complexes. This change produces the relative drop in $\sigma_{0}(T)$ in the vicinity of $T_{\sigma}$. For $T>T_{\sigma}$, the conduction band is being filled with the released electrons and the Fermi level rises until a metal-like state is reached (see schematics in Supplemental Fig. S5 [43]).

When the heated excited films are cooled, their hightemperature behavior is an outcome from the electrons' release and trapping back to the $\left(\mathrm{V}_{\mathrm{O}}^{+2}-\mathrm{Ti}^{3+}\right)^{+}$complexes. This picture contrasts with the frequently discussed motion of oxygen vacancies and the related hopping of the large polarons. Indeed, the conductivity is directly proportional to the concentration $n$ of free charge carriers, which, in turn, results from the release and trapping of the electrons here. Because of the activation character of the release, the concentration is $n \propto \exp \left(-E_{n} / k T\right)$, where the energy $E_{n}$ characterizes both the release or trapping and the changes of the Fermi level. Thus the magnitude of $E_{n}$ is between the electron binding energy, $E_{b} \approx 0.2-0.4 \mathrm{eV}$, and half of the band gap, $E_{g} / 2 \approx 1.7-1.9 \mathrm{eV}[46,36]$. Therefore, the temperature-dependent semiconductor-type conductivity $\left[\sigma \propto \exp \left(-E_{n} / k T\right)\right]$ can simply mimic the large-polaron hopping with $E_{h} \approx 0.8-0.95 \mathrm{eV}$ (Table II). The suggested scenario easily bridges the high-temperature semiconductortype conductivity in the oxygen-deficient 2-10-Pa films with the semiconductor-type conductivity in the strongly oxygendeficient $0.1-\mathrm{Pa}$ film in the broad range of temperatures. This scenario also explains the difference between the smallpolaron energies in the virgin and excited states (Table II). Compared to the virgin state, the released free electrons partly screen the ferroelectric polarization in the excited state. Because of the polarization screening, the polaron binding energy decreases [48] and, correspondingly, smaller hopping energy is observed in the excited state.

We note that the out-of-plane oxygen vacancies are formed under action of the substrate-imposed compressive strain and, therefore, their positions and charges are elastically stabilized in the films. Migration of such elastically stabilized vacancies is unlikely. We argue that also under applied electric field, the oxygen-vacancy-related electronic release and trapping can take place, which imitates the widely assumed migration of the mobile oxygen vacancies. Namely, in the $\left(\mathrm{V}_{\mathrm{O}}^{+2}-\mathrm{Ti}^{3+}\right)^{+}$ complexes, the electron binding energy is $\sim 0.2-0.3 \mathrm{eV}$, which facilitates electronic release under a certain electric field. The field-stimulated release and drift of the electrons may have the appearance of motion and accumulation of the positively charged oxygen vacancies. Likewise, when excess electrons are injected from an electrode into the film, some of them can be trapped by the $\left(\mathrm{V}_{\mathrm{O}}^{+2}-\mathrm{Ti}^{3+}\right)^{+}$and/or $\mathrm{V}_{\mathrm{O}}^{+2}$ defects, thus diminishing the positive charge. This trapping looks like a drift of the positively charged oxygen vacancies away from the electrode. Similar to applying electric field, light illumination also can stimulate the electronic release and trapping, which may give the feeling of mobile oxygen vacancies.

The peculiar electronic processes, which are associated with the strain-stabilized immobile oxygen vacancies, require further studies. Theoretical modeling of the formation and structure of the oxygen vacancies and the related defects, as well as of the associated transport phenomena in ferroelectrics, particularly in epitaxial films, would be invaluable.

\section{CONCLUSIONS}

Small-signal conductivity was studied by impedance analysis in the broad ranges of frequency $f=1-10^{6} \mathrm{~Hz}$ and temperature $T=10-720 \mathrm{~K}$ in cube-on-cube-type epitaxial BTO films, possessing different in-plane strains and degrees of oxygen deficiency. The low-temperature hopping of small polarons and high-temperature semiconductor- to metal-type behavior were detected. The small polarons were ascribed to electrons self-trapped at individual $\mathrm{Ti}$ atoms in the tensilestrained stoichiometric film and to electrons trapped at $\mathrm{Ti}$ next to the doubly charged oxygen vacancies in the compressively strained oxygen-deficient films. The thermally activated release of the trapped electrons was shown to lead to the conduction-band transport. It was also suggested that the electronic release can mimic the motion of the oxygen vacancies, while these vacancies are strain stabilized and immobile.

\section{ACKNOWLEDGMENTS}

The authors acknowledge support from the Czech Science Foundation (Grant No. 19-09671S) and the European Structural and Investment Funds and the Ministry of Education, Youth and Sports of the Czech Republic through Programme "Research, Development and Education" (Project No. SOLID21-CZ.02.1.01/0.0/0.0/16_019/0000760). The authors are grateful to T. Kocourek for sample preparation and to J. Peräntie for structural analysis.
[1] M. E. Lines and A. M. Glass, Principles and Applications of Ferroelectrics and Related Materials (Clarendon Press, Oxford, 2004).

[2] K. Uchino, Ferroelectric Devices (Marcel Dekker, New York, 2000)
[3] J. F. Scott, Ferroelectric Memories (Springer-Verlag, Berlin, 2000).

[4] N. Setter et al., Ferroelectric thin films: Review of materials, properties, and applications, J. Appl. Phys. 100, 051606 (2006). 
[5] M. Dawber, K. M. Rabe, and J. F. Scott, Physics of thin-film ferroelectric oxides, Rev. Mod. Phys. 77, 1083 (2005).

[6] Physics of Ferroelectrics: A Modern Perspective, edited by K. M. Rabe, C. H. Ahn, and J.-M. Triscone (Springer-Verlag, Berlin, 2007).

[7] L. W. Martin and A. M. Rappe, Thin-film ferroelectric materials and their applications, Nat. Rev. Mater. 2, 16087 (2016).

[8] Ferroelectric Materials for Energy Applications, edited by H. Huang and J. F. Scott (Wiley-VCH Verlag, Weinheim, Germany, 2018).

[9] H. Arend and L. Kihlborg, Phase composition of reduced and reoxidized barium titanate, J. Am. Ceram. Soc. 52, 68 (1969).

[10] T. Kolodiazhnyi, A. Petric, M. Niewczas, C. Bridges, A. SafaSefat, and J. E. Greedan, Thermoelectric power, Hall effect, and mobility of $n$-type $\mathrm{BaTiO}_{3}$, Phys. Rev. B 68, 085205 (2003).

[11] T. Kolodiazhnyi, Insulator-metal transition and anomalous sign reversal of the dominant charge carriers in perovskite $\mathrm{BaTiO}_{3-\delta}$, Phys. Rev. B 78, 045107 (2008).

[12] J. Hwang, T. Kolodiazhnyi, J. Yang, and M. Couillard, Doping and temperature-dependent optical properties of oxygenreduced $\mathrm{BaTiO}_{3-\delta}$, Phys. Rev. B 82, 214109 (2010).

[13] H. Ihrig and D. Hennings, Electrical transport properties of $n$-type $\mathrm{BaTiO}_{3}$, Phys. Rev. B 17, 4593 (1978), and references therein.

[14] J. P. Boyeaux and F. M. Michel-Calendini, Small polaron interpretation of $\mathrm{BaTiO}_{3}$ transport properties from drift mobility measurements, J. Phys. C: Solid State Phys. 12, 545 (1979).

[15] E. Iguchi, N. Kubota, T. Nakamori, N. Yamamoto, and K. J. Lee, Polaronic conduction in $n$-type $\mathrm{BaTiO}_{3}$ doped with $\mathrm{La}_{2} \mathrm{O}_{3}$ or $\mathrm{Gd}_{2} \mathrm{O}_{3}$, Phys. Rev. B 43, 8646 (1991).

[16] I. P. Raevski, S. M. Maksimov, A. V. Fisenko, S. A. Prosandeyev, I. A. Osipenko, and P. F. Tarasenko, Study of intrinsic point defects in oxides of the perovskite family: II. Experiment, J. Phys.: Condens. Matter 10, 8015 (1998).

[17] R. Waser, T. Baiatu, and K.-H. Hardtl, dc electrical degradation of perovskite-type titanates: I, ceramics, J. Am. Ceram. Soc. 73, 1645 (1990).

[18] C. Ang, Z. Yu, and L. E. Cross, Oxygen-vacancy-related lowfrequency dielectric relaxation and electrical conduction in Bi: $\mathrm{SrTiO}_{3}$, Phys. Rev. B 62, 228 (2000).

[19] B. S. Kang, S. K. Choi, and C. H. Park, Diffuse dielectric anomaly in perovskite-type ferroelectric oxides in the temperature range of 400-700 ${ }^{\circ}$ C, J. Appl. Phys. 94, 1904 (2003).

[20] R. Meyer, R. Liedtke, and R. Waser, Oxygen vacancy migration and time-dependent leakage current behavior of $\mathrm{Ba}_{0.3} \mathrm{Sr}_{0.7} \mathrm{TiO}_{3}$ thin films, Appl. Phys. Lett. 86, 112904 (2005).

[21] A. G. Boni, I. Pintilie, L. Pintilie, D. Preziosi, H. Deniz, and M. Alexe, Electronic transport in $(\mathrm{La}, \mathrm{Sr}) \mathrm{MnO}_{3}$-ferroelectric(La,Sr) $\mathrm{MnO}_{3}$ epitaxial structures, J. Appl. Phys. 113, 224103 (2013).

[22] R. A. Maier and C. A. Randall, Low temperature ionic conductivity of an acceptor-doped perovskite: II. Impedance of single-crystal $\mathrm{BaTiO}_{3}$, J. Am. Ceram. Soc. 99, 3360 (2016).

[23] Y. Heo, D. Kan, Y. Shimakawa, and J. Seidel, Resistive switching properties of epitaxial $\mathrm{BaTiO}_{3-\delta}$ thin films tuned by aftergrowth oxygen cooling pressure, Phys. Chem. Chem. Phys. 18, 197 (2016).
[24] M. Li, J. Zhou, X. Jing, M. Zeng, S. Wu, J. Gao, Z. Zhang, X. Gao, X. Lu, J.-M. Liu, and M. Alexe, Controlling resistance switching polarities of epitaxial $\mathrm{BaTiO}_{3}$ films by mediation of ferroelectricity and oxygen vacancies, Adv. Electron. Mater. 1, 1500069 (2015).

[25] N. Du, N. Manjunath, Y. Li, S. Menzel, E. Linn, R. Waser, T. You, D. Bürger, I. Skorupa, D. Walczyk, C. Walczyk, O. G. Schmidt, and H. Schmidt, Field-Driven Hopping Transport of Oxygen Vacancies in Memristive Oxide Switches with Interface-Mediated Resistive Switching, Phys. Rev. Appl. 10, 054025 (2018).

[26] T. Zhao, F. Chen, H. Lu, G. Yang, and Z. Chen, Thickness and oxygen pressure dependent structural characteristics of $\mathrm{BaTiO}_{3}$ thin films grown by laser molecular beam epitaxy, J. Appl. Phys. 87, 7442 (2000).

[27] S. B. Mia, C. L. Jia, T. Heeg, O. Trithaveesak, J. Schubert, and K. Urban, Heterostructures of $\mathrm{BaTiO}_{3}$ bilayer films grown on $\mathrm{SrTiO}_{3}(001)$ under different oxygen pressures, J. Cryst. Growth 283, 425 (2005).

[28] Y. L. Zhu, S. J. Zheng, D. Chen, and X. L. Ma, Microstructure tuning of epitaxial $\mathrm{BaTiO}_{3-x}$ thin films grown using laser molecular beam epitaxy by varying the oxygen pressure, Thin Solid Films 518, 3669 (2010).

[29] A. P. Chen, F. Khatkhatay, W. Zhang, C. Jacob, L. Jiao, and H. Wang, Strong oxygen pressure dependence of ferroelectricity in $\mathrm{BaTiO}_{3} / \mathrm{SrRuO}_{3} / \mathrm{SrTiO}_{3}$ epitaxial heterostructures, J. Appl. Phys. 114, 124101 (2013).

[30] J. Lyu, S. Estandía, J. Gazquez, M. F. Chisholm, I. Fina, N. Dix, J. Fontcuberta, and F. Sánchez, Control of polar orientation and lattice strain in epitaxial $\mathrm{BaTiO}_{3}$ films on silicon, ACS Appl. Mater. Interfaces 10, 25529 (2018).

[31] J. N. Baker, P. C. Bowes, J. S. Harris, and D. L. Irving, Mechanisms governing metal vacancy formation in $\mathrm{BaTiO}_{3}$ and $\mathrm{SrTiO}_{3}$, J. Appl. Phys. 124, 114101 (2018).

[32] A. Herklotz, D. Lee, E.-J. Guo, T. L. Meyer, J. R. Petrie, and H. N. Lee, Strain coupling of oxygen nonstoichiometry in perovskite thin films, J. Phys.: Condens. Matter 29, 493001 (2017).

[33] U. Aschauer and N. A. Spaldin, Interplay between strain, defect charge state, and functionality in complex oxides, Appl. Phys. Lett. 109, 031901 (2016).

[34] J. Xi, H. Xu, Y. Zhang, and W. J. Weber, Strain effects on oxygen vacancy energetics in $\mathrm{KTaO}_{3}$, Phys. Chem. Chem. Phys. 19, 6264 (2017).

[35] M. Tyunina, O. Pacherova, J. Peräntie, M. Savinov, M. Jelinek, H. Jantunen, and A. Dejneka, Perovskite ferroelectric tuned by thermal strain, Sci. Rep. 9, 3677 (2019).

[36] M. Tyunina, D. Chvostova, and A. Dejneka, Optical revelation of defects in epitaxial barium titanate films, Phys. Chem. Chem. Phys. 21, 7874 (2019).

[37] O. Dieguez, S. Tinte, A. Antons, C. Bungaro, J. B. Neaton, K. M. Rabe, and D. Vanderbilt, $A b$ initio study of the phase diagram of epitaxial $\mathrm{BaTiO}_{3}$, Phys. Rev. B 69, 212101 (2004).

[38] V. B. Shirokov, Yu. I. Yuzyuk, B. Dkhil, and V. V. Lemanov, Phenomenological theory of phase transitions in epitaxial $\mathrm{BaTiO}_{3}$ thin films, Phys. Rev. B 75, 224116 (2007).

[39] M. Tyunina and J. Levoska, Application of the interface capacitance model to thin-film relaxors and ferroelectrics, Appl. Phys. Lett. 88, 262904 (2006). 
[40] M. Tyunina, Size effects and dielectric behaviour in ferroelectric heterostructures, J. Phys.: Condens. Matter 18, 5725 (2006).

[41] M. Tyunina, M. Plekh, M. Antonova, and A. Kalvane, Ferroelectric transitions in epitaxial $\mathrm{Pb}_{0.5} \mathrm{Sr}_{0.5} \mathrm{TiO}_{3}$ films studied by dielectric analysis, Phys. Rev. B 84, 224105 (2011).

[42] M. Tyunina, B. Malic, M. Plekh, and M. Kosec, Dielectric response of $\mathrm{BaTiO}_{3}$ thin film with grain size at nanometer scale, J. Am. Ceram. Soc. 95, 1333 (2012).

[43] See Supplemental Material at http://link.aps.org/supplemental/ 10.1103/PhysRevB.101.094106 for details of impedance analysis.

[44] A. K. Jonscher, Universal Relaxation Law (Chelsea Dielectrics Press, London, 1995).
[45] A. Ghosh, Frequency-dependent conductivity in bismuthvanadate glassy semiconductors, Phys. Rev. B 41, 1479 (1990).

[46] A. Janotti, J. B. Varley, M. Choi, and C. G. Van de Walle, Vacancies and small polarons in $\mathrm{SrTiO}_{3}$, Phys. Rev. B 90, 085202 (2014).

[47] V. E. Alexandrov, E. A. Kotomin, J. Maier, and R. A. Evarestov, First-principles study of bulk and surface oxygen vacancies in $\mathrm{SrTiO}_{3}$ crystal, Eur. Phys. J. B 72, 53 (2009).

[48] T. Xu, T. Shimada, Y. Araki, M. Mori, G. Fujimoto, J. Wang, T.-Y. Zhang, and T. Kitamura, Electron engineering of metallic multiferroic polarons in epitaxial $\mathrm{BaTiO}_{3}$, npj Comput. Mater. 5, 23 (2019). 